\title{
Interference-based Capacity Analysis in CDMA Cellular Systems
}

\author{
Joonhwan Kim*, Young-June Choi ${ }^{\dagger}$, and Saewoong Bahk ${ }^{\dagger}$ \\ ${ }^{*}$ GCT Semiconductor, Seoul 150-712, Korea \\ ${ }^{\dagger}$ School of Electrical Engineering \& Computer Science, Seoul National University, Korea \\ E-mail: *kjh@gctsemi.com, ${ }^{\dagger}\{$ yjchoi,sbahk $\} @$ netlab.snu.ac.kr
}

\begin{abstract}
In multiple cellular CDMA systems, the channel capacity heavily depends upon the interference caused by the signal power of other users. In this paper, we exactly quantify the cell capacity by focusing on not only the number of users but also the location of active users. To inspect the effect of multicell CDMA environments, we model three $1 / 6$ cells divided by $60^{\circ}$ sector antennas and analyze the increased interference when a user is added to the unit of cell. Based on the capacity analysis, we propose a new call admission control scheme that uses the location information of users. Whenever a new user arrives, our scheme checks whether the admittance of that user can occur within the cell capacity. If the user is far away from the base station, it will have less chance of acceptance because it creates more interference, resulting in reduced capacity. Our scheme allows more users to enter the network if they generate less interference. Therefore it has the effect of increased system capacity without sacrificing the QoS of all the other active users. Through simulations we show that the performance is improved by 10 to $20 \%$ in terms of the number of active users that can be accomodated.
\end{abstract}

\section{INTRODUCTION}

A mobile user in FDMA and TDMA systems uses a dedicated channel to communicate with the base station (BS). As there is no resource sharing among mobile users, a user's call quality is not affected by the other users at all. A user in CDMA systems, however, shares the same frequency band with the others. Therefore the quality of a call is influenced by the other users' signal power, which is considered as the interference to itself. We can use power control to maintain the appropriate signal to interference ratio (SIR), thereby keeping the call quality [2], [3], [8]. Especially the transmitter must control the power at the uplink channel to provide the same SNR for all users regardless of the noise level, which consists of intercell interference, intracell interference and thermal noise.

The TDMA or FDMA systems can serve a new user if there is a free time slot or idle frequency band. But it is not easy to decide whether the new call can be admitted into the CDMA systems without considering the noise generated by the new call, especially when the channels are fully utilized. This is because the signal power of a mobile user becomes noise for others in the CDMA systems. So call admission control $(\mathrm{CAC})$ is required to maintain the call quality of existing users and also power control to prevent system outage [1], [4]. Their works have mainly considered only the average number of mobile users by counting intercell interference.

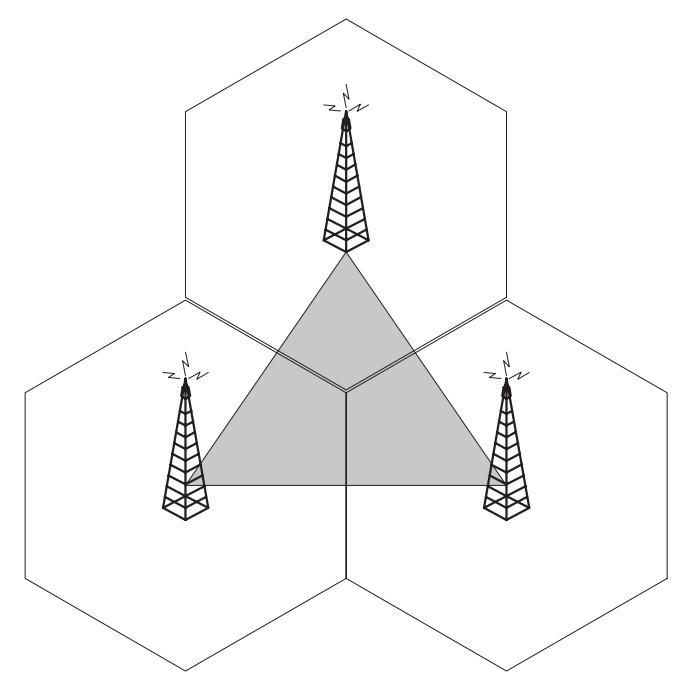

Fig. 1. System Model.

While the maximum number of users that can be served is bounded deterministically in the FDMA/TDMA systems, it is not true in the CDMA systems because of interference effect. So we need to consider the states of other neighboring cells to use channel resource effectively for multiple cellular CDMA environments. In [5], Kim investigated the average value of intercell interference.

As the uplink channel limits coverage [4], we focus on uplink communication in order to enhance system capacity. We assume the channel condition is influenced by path loss only. So the transmission power of a mobile user near the cell boundary is much higher than that of a user close to the BS. Using this characteristic, the system will be able to admit more mobile users near the BS by blocking cell boundary users. This results in higher number of active users within the cell. To achieve this, we inevitably sacrifice fairness between near and far users. In this paper we will consider a new CAC that uses the information about a user's distance from the corresponding BS. Before doing this we will more exactly quantify channel resource of CDMA in multiple cells.

We can classify arrival calls as new calls and handoff calls. Handoff calls have priority over new calls as handoff call dropping badly affects the QoS of users. So handoff calls are prioritized to reduce handoff call dropping [7]. We do not 
consider the mobility of users inside the cell. We assume one type of user traffic, i.e., voice in the network. Then we try to maximize the number of mobile users by selective admission of new calls according to the signal power that is reversely proportional to the distance from the BS.

We organize the remainder of our paper as follows. In Section II we will present the system model which uses $60^{\circ}$ sector antennas and quantify the channel resource in Section III. In Section IV we will present two level CAC algorithm to use the analysis result of Section III. We will show simulation results in Section V, and finally concludes in Section VI.

\section{SYSTEM MODEL}

\section{A. System with $60^{\circ}$ sector antennas}

Sector antennas are widely used for many BSs. Using $60^{\circ}$ sector antennas we can express the system model as shown in Fig. 1. Theoretically the gain of a $60^{\circ}$ sector antenna is six times larger than that of an omni directional antenna. By defining the unit region, we can analyze this antenna more easily than an omni directional antenna or a $120^{\circ}$ antenna. As there are three $1 / 6$ cells divided by $60^{\circ}$ sector antennas, we call this unit region $3 \frac{1}{6}$ cell indicated as the shaded area in Fig. $1[6]$.

As the interference caused by users outside of the $3 \frac{1}{6}$ region is relatively small when compared with that inside this region, we assume that relavant intercell interference sources are limited to the shaded area in Fig. 1.

\section{B. System Description}

We define the state as the number of users at an infinitesimal region (IR) in Fig. 2 and assume that there are $N$ IRs in each sector. For the system model we define the following parameters.

- $d_{k j}$ : the distance from BS $k$ to $j$

- $s_{j}$ : the area of IR $j$

- $x_{j}$ : the number of users in IR $j$

- $\mathbf{X}=\left(x_{1}, \cdots, x_{3 N}\right):$ the state vector in the unit region

where $k$ and $j$ indicate BS and IR with the ranges of $1 \leq k \leq 3$ and $0 \leq j \leq 3 N$ respectively. There are $3 N$ elements in the state vector where $\left(x_{1}, \cdots x_{N}\right),\left(x_{N+1}, \cdots x_{2 N}\right)$, and $\left(x_{2 N+1}, \cdots x_{3 N}\right)$ represent the number of users at $N$ IRs in cells 1-3 respectively.

Let $I_{o c, k}$ be the other cell's interference received at BS $k$. From the assumption that the channel is influenced only by path loss, $I_{o c, k}$ can be written as

$$
I_{o c, k}=\sum_{j \notin C e l l-k} \alpha \cdot\left(d_{k j}\right)^{n} \cdot P_{j} \cdot x_{j}
$$

where $\alpha, n$, and $P_{j}$ indicate a constant, the path loss exponent, and the transmitted power by a user in IR $j . I_{o c, k}$ reflects on only the power transmitted by the users in the other two sectors. The power to be transmitted at antenna $j, P_{j}$, must be controlled to receive the same power conversely. It can be written as

$$
P_{j}=q_{k} / \alpha \cdot\left(d_{k j}\right)^{n}
$$

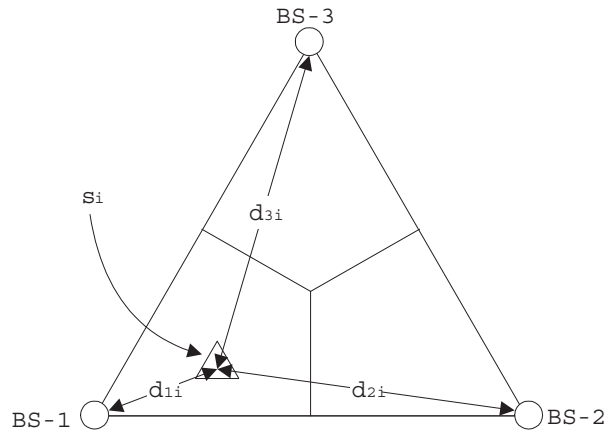

Fig. 2. Infinitesimal region in $3 \frac{1}{6}$ cell.

where $q_{k}$ is the received power at a sector antenna in BS $k$ that is optimized for the following.

$$
q_{k}^{*}=\frac{I_{o c, k}+\eta_{0}}{1 / S I R_{\min }+1-N_{k}}=\frac{\operatorname{SIR}_{\min }\left(I_{o c, k}+\eta_{0}\right)}{1-S I R_{\min }\left(N_{k}-1\right)} .
$$

To solve these equations, we define

$$
\begin{array}{r}
\mathbf{B}_{k l}=\sum_{j=(l-1) N+1}^{l N} x_{j} \cdot\left(\frac{d_{k j}}{d_{l j}}\right)^{n} \\
\mathbf{C}_{k l}=\frac{\mathbf{B}_{k l} \cdot \hat{\Gamma}}{1-\hat{\Gamma}\left(N_{l}-1\right)} .
\end{array}
$$

$I_{o c, k}$ can be represented by (3) and (4).

$$
\begin{aligned}
& I_{o c, 1}=\mathrm{B}_{12} q_{2}^{*}+\mathrm{B}_{13} q_{3}^{*} \\
& I_{o c, 2}=\mathrm{B}_{21} q_{1}^{*}+\mathrm{B}_{23} q_{3}^{*} \\
& I_{o c, 3}=\mathrm{B}_{31} q_{1}^{*}+\mathrm{B}_{32} q_{3}^{*} .
\end{aligned}
$$

From these, we can obtain

$$
\left(\begin{array}{c}
I_{o c, 1} \\
I_{o c, 2} \\
I_{o c, 3}
\end{array}\right)=\left(\begin{array}{ccc}
1 & -\mathbf{C}_{12} & -\mathbf{C}_{13} \\
-\mathbf{C}_{21} & 1 & -\mathbf{C}_{23} \\
-\mathbf{C}_{31} & -\mathbf{C}_{32} & 1
\end{array}\right)^{-1}\left(\begin{array}{c}
\left(\mathbf{C}_{12}+\mathbf{C}_{13}\right) \cdot \eta_{0} \\
\left(\mathbf{C}_{21}+\mathbf{C}_{23}\right) \cdot \eta_{0} \\
\left(\mathbf{C}_{31}+\mathbf{C}_{32}\right) \cdot \eta_{0}
\end{array}\right)
$$

\section{ANALYSis of CDMA RESOURCE}

In this section we will quantify the resource in multiple CDMA cells. The total interference received at a BS in the unit region, $\mathbf{I}_{\boldsymbol{\Sigma}}$, is the sum of three $I_{o c, k}$ 's in (7).

$$
\begin{aligned}
& \mathbf{I}_{\boldsymbol{\Sigma}}=I_{o c, 1}+I_{o c, 2}+I_{o c, 3}
\end{aligned}
$$

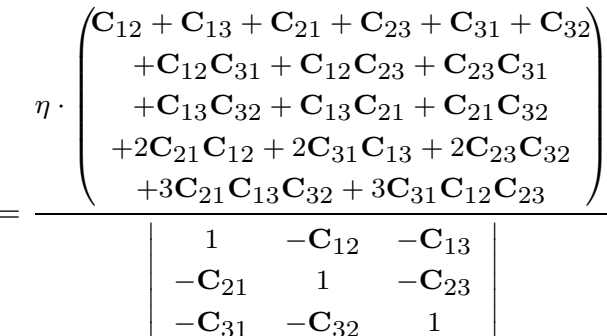

$$
\begin{aligned}
& =\frac{\mathbf{N}}{\mathbf{D}} \cdot \eta_{0}
\end{aligned}
$$


If a user is admitted into cell 1 , the increased interference, $\widetilde{\mathbf{I}_{\boldsymbol{\Sigma}}}$, is given by

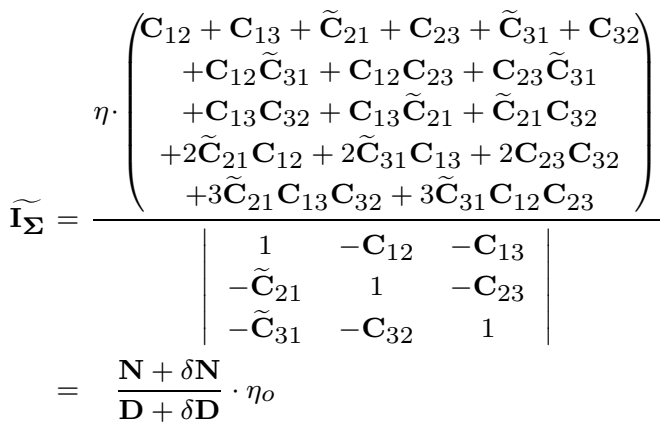

where

$$
\begin{aligned}
\delta \mathbf{N} & =\left(1+\mathbf{C}_{13}+\mathbf{C}_{32}+2 \mathbf{C}_{12}+3 \mathbf{C}_{13} \mathbf{C}_{32}\right)\left(\widetilde{\mathbf{C}}_{21}-\mathbf{C}_{21}\right) \\
& +\left(1+\mathbf{C}_{12}+\mathbf{C}_{23}+2 \mathbf{C}_{13}+3 \mathbf{C}_{12} \mathbf{C}_{23}\right)\left(\widetilde{\mathbf{C}}_{31}-\mathbf{C}_{31}\right) \\
\delta \mathbf{D} & =\left(\mathbf{C}_{12}+\mathbf{C}_{13} \cdot \mathbf{C}_{32}\right) \cdot\left(\mathbf{C}_{21}-\widetilde{\mathbf{C}}_{21}\right) \\
& +\left(\mathbf{C}_{13}+\mathbf{C}_{12} \cdot \mathbf{C}_{23}\right) \cdot\left(\mathbf{C}_{31}-\widetilde{\mathbf{C}}_{31}\right)
\end{aligned}
$$

Then the increased interference $\Delta \widetilde{\mathbf{I}_{\Sigma}}$ is given by

$$
\begin{aligned}
\Delta \widetilde{\mathbf{I}_{\boldsymbol{\Sigma}}} & =\widetilde{\mathbf{I}_{\boldsymbol{\Sigma}}}-\mathbf{I}_{\boldsymbol{\Sigma}} \\
& =\left(\frac{\mathbf{N}+\delta \mathbf{N}}{\mathbf{D}+\delta \mathbf{D}}-\frac{\mathbf{N}}{\mathbf{D}}\right) \cdot \eta_{0} \\
& =\left(\frac{\mathbf{D} \cdot \delta \mathbf{N}-\mathbf{N} \cdot \delta \mathbf{D}}{\mathbf{D}(\mathbf{D}+\delta \mathbf{D})}\right) \cdot \eta_{0}
\end{aligned}
$$

When a user is added to cell 1 , the interference at the two other cells also increases. Let $\widetilde{\mathbf{C}}_{k 1}$ be the adjusted value of $\mathbf{C}_{k 1}$ to maintain $S I R_{\min }$ of cell $k$. Then the increased value is given by

$$
\begin{aligned}
\Delta \widetilde{\mathbf{C}}_{i 1} & =\widetilde{\mathbf{C}}_{i 1}-\mathbf{C}_{i 1} \\
& =\frac{\widetilde{\mathbf{B}}_{i 1}}{\hat{N}-N_{1}-1}-\frac{\mathbf{B}_{i 1}}{\hat{N}-N_{1}}=\frac{\mathbf{B}_{i 1}+\Delta \widetilde{\mathbf{B}}_{i 1}}{\hat{N}-N_{1}-1}-\frac{\mathbf{B}_{i 1}}{\hat{N}-N_{1}} \\
& =\Delta \widehat{\mathbf{C}}_{i 1}+\frac{1}{\Gamma-N_{1}-1} \cdot \Delta \widetilde{\mathbf{B}}_{i 1}
\end{aligned}
$$

where $\Delta \widehat{\mathbf{C}}_{i 1}$ and $\Delta \widetilde{\mathbf{B}}_{i 1}$ is

$$
\begin{aligned}
\Delta \widehat{\mathbf{C}}_{i 1} & =\frac{\widehat{\mathbf{B}}_{i 1}}{\hat{N}-N_{1}-1}-\frac{\mathbf{B}_{i 1}}{\hat{N}-N_{1}} \\
& =\mathbf{B}_{i 1}\left(\frac{1}{\hat{N}-N_{1}-1}-\frac{1}{\hat{N}-N_{1}}\right) \\
\Delta \widetilde{\mathbf{B}}_{i 1} & =\widetilde{\mathbf{B}}_{i 1}-\mathbf{B}_{i 1}=\left(\frac{d_{i s}}{d_{1 s}}\right)^{n}
\end{aligned}
$$

Here ', represents the varied value when a user is added, , , indicates the varied value when the user is near the BS (i.e., small intercell interference), and ' $\Delta^{\prime}$ ' represents the difference after the user is added. As shown in (16), $\Delta \widetilde{\mathbf{B}}_{i 1}$ is a fuction of the user's distance from the corresponding BS $d_{1 s}$ and from neighbor BSs $d_{i s}$ 's. Then $\Delta \widetilde{\mathbf{I}_{\Sigma}}$ can be expressed as

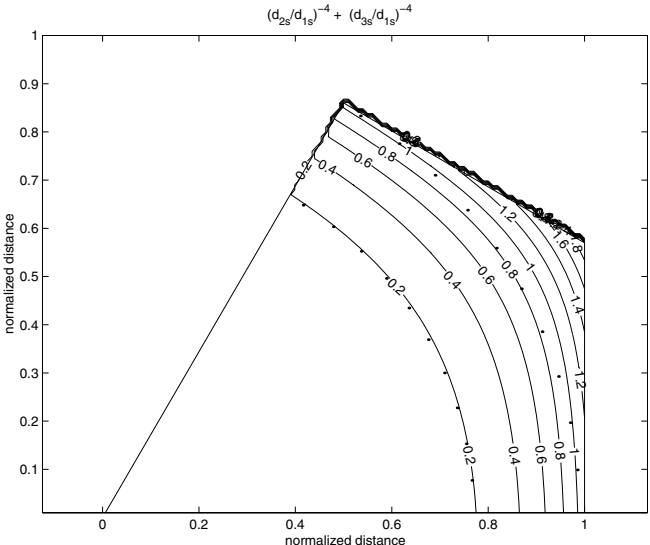

Fig. 3. User location vs. $\left(\frac{d_{2 i}}{d_{1 i}}\right)^{n}+\left(\frac{d_{3 i}}{d_{1 i}}\right)^{n}$.

the following.

$$
\begin{aligned}
\Delta \widetilde{\mathbf{I}_{\boldsymbol{\Sigma}}}= & \mathbf{U} \frac{\mathbf{U}_{n 1} \Delta \widetilde{\mathbf{C}}_{21}+\mathbf{U}_{n 2} \Delta \widetilde{\mathbf{C}}_{31}}{\mathbf{U}_{d 1} \Delta \widetilde{\mathbf{C}}_{21}+\mathbf{U}_{d 2} \Delta \widetilde{\mathbf{C}}_{31}+\mathbf{U}_{d 3}} \\
= & \mathbf{U} \frac{\left(\begin{array}{l}
\mathbf{U}_{n 2}\left(\mathbf{B}_{21}\left(\frac{1}{\hat{N}-N_{1}-1}-\frac{1}{\hat{N}-N_{1}}\right)+\frac{1}{\Gamma-N_{1}-1}\left(\frac{d_{2 i}}{d_{i i}}\right)^{n}\right) \\
+\mathbf{U}_{n 3}\left(\mathbf{B}_{31}\left(\frac{1}{\hat{N}-N_{1}-1}-\frac{1}{\hat{N}-N_{1}}\right)+\frac{1}{\Gamma-N_{1}-1}\left(\frac{d_{3 i}}{d_{1 i}}\right)^{n}\right)
\end{array}\right)}{\left(\begin{array}{l}
\mathbf{U}_{d 1} \\
-\mathbf{U}_{d 2}\left(\mathbf{B}_{21}\left(\frac{1}{\hat{N}-N_{1}-1}-\frac{1}{\hat{N}-N_{1}}\right)+\frac{1}{\Gamma-N_{1}-1}\left(\frac{d_{2 i}}{d_{1 i}}\right)^{n}\right) \\
-\mathbf{U}_{d 3}\left(\mathbf{B}_{31}\left(\frac{1}{\hat{N}-N_{1}-1}-\frac{1}{\hat{N}-N_{1}}\right)+\frac{1}{\Gamma-N_{1}-1}\left(\frac{d_{3 i}}{d_{1 i}}\right)^{n}\right)
\end{array}\right)}
\end{aligned}
$$

where

$$
\begin{aligned}
& \mathbf{U}_{n 2}=1+\mathbf{C}_{12}+\mathbf{C}_{32}+\mathbf{C}_{13} \mathbf{C}_{32}+\left(\mathbf{C}_{12}-\mathbf{C}_{13}\right) \mathbf{C}_{31} \\
& \mathbf{U}_{n 3}=1+\mathbf{C}_{23}+\mathbf{C}_{13}+\mathbf{C}_{12} \mathbf{C}_{23}+\left(\mathbf{C}_{13}-\mathbf{C}_{12}\right) \mathbf{C}_{21} \\
& \mathbf{U}_{d 2}=\mathbf{C}_{12}+\mathbf{C}_{13} \mathbf{C}_{32} \\
& \mathbf{U}_{d 3}=\mathbf{C}_{12} \mathbf{C}_{23}+\mathbf{C}_{13} \\
& \mathbf{U}_{d 1}=1-\left(\mathbf{C}_{21} \mathbf{C}_{12}+\mathbf{C}_{23} \mathbf{C}_{32}+\mathbf{C}_{31} \mathbf{C}_{13}+\mathbf{C}_{21} \mathbf{C}_{13} \mathbf{C}_{32}+\mathbf{C}_{31} \mathbf{C}_{12} \mathbf{C}_{23}\right)
\end{aligned}
$$

When a user is added, we can divide the increased components of $\Delta \widetilde{\mathbf{I}_{\Sigma}}$ into two parts. One is an increased part regardless of the user's location and the other is an increased part depending on it. In (17), $\left(\frac{d_{2 i}}{d_{1 i}}\right)^{n}$ and $\left(\frac{d_{3 i}}{d_{1 i}}\right)^{n}$ are the resulting user's relative locations and $\frac{1}{\hat{N}-N_{1}-1}$ is the added result of a user. Now we assume that $N$ users are in a sector and they are uniformly distributed within the sector. The expectation value of $\mathbf{B}_{12}$ is given by

$$
\begin{array}{r}
E\left[\mathbf{B}_{12}\right]=E\left[\sum_{j=N+1}^{2 N} x_{j} \cdot\left(\frac{d_{1 j}}{d_{2 j}}\right)^{n}\right] \\
=\sum_{j=N+1}^{2 N} x_{j} \cdot E\left[\left(\frac{d_{1 j}}{d_{2 j}}\right)^{n}\right]=N \cdot E\left[\left(\frac{d_{1 j}}{d_{2 j}}\right)^{n}\right] .
\end{array}
$$

If we let $\mathrm{E}$ be $E\left[\left(d_{1 j} / d_{2 j}\right)^{n}\right], E\left[\mathbf{B}_{12}\right]=E\left[\mathbf{B}_{13}\right]=E\left[\mathbf{B}_{21}\right]=$ $E\left[\mathbf{B}_{23}\right]=E\left[\mathbf{B}_{31}\right]=E\left[\mathbf{B}_{32}\right]=N \cdot \mathbf{E}$, then the expectation value of $\mathbf{C}_{\mathbf{k l}}$ can be written as

$$
E\left[\mathbf{C}_{k l}\right]=\frac{\mathrm{E} \cdot N}{\hat{N}-N}
$$




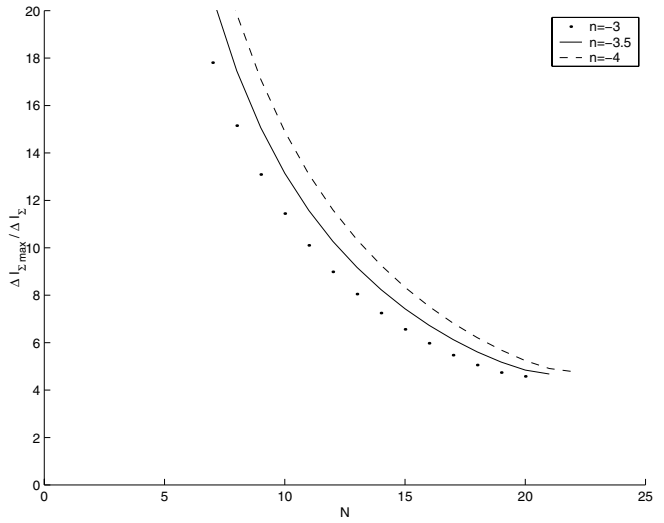

Fig. 4. Average number of users and Path loss exponents vs. $\Delta \mathbf{I}_{\mathbf{\Sigma}} \max / \Delta \widehat{\mathbf{I}_{\boldsymbol{\Sigma}}}$.

E can be given by an integral over the area of IRs as the following.

$$
\begin{aligned}
E\left[\left(\frac{d_{1 j}}{d_{2 j}}\right)^{n}\right] & =\lim _{\substack{N \rightarrow \infty \\
s_{j} \rightarrow 0}} \sum_{j=N+1}^{2 N} \frac{s_{j}}{S} \cdot\left(\frac{d_{1 j}}{d_{2 j}}\right)^{n} \\
& =\int_{1}^{\frac{3}{2}} \int_{0}^{\frac{1}{\sqrt{3}} x}\left(\frac{\sqrt{x^{2}+y^{2}}}{\sqrt{(x-2)^{2}+y^{2}}}\right)^{n} d y d x
\end{aligned}
$$

Using (21), we obtain $E=0.219,0.188,0.164$ for $n=-3,-3.5$, -4 respectively. From the above we can rewrite $\Delta \widetilde{\mathbf{I}_{\boldsymbol{\Sigma}}}$ as the following.

$$
\Delta \widetilde{\mathbf{I}_{\boldsymbol{\Sigma}}}=\frac{\mathbf{V}_{n 4}+\mathbf{V}_{n 5} \cdot\left(\left(\frac{d_{2 i}}{d_{1 i}}\right)^{n}+\left(\frac{d_{3 i}}{d_{1 i}}\right)^{n}\right)}{\mathbf{V}_{d 4}-\mathbf{V}_{d 5} \cdot\left(\left(\frac{d_{2 i}}{d_{1 i}}\right)^{n}+\left(\frac{d_{3 i}}{d_{1 i}}\right)^{n}\right)}
$$

where

$$
\begin{aligned}
\mathbf{V}_{n 4} & =\frac{2\left(E\left[\mathbf{C}_{k l}\right]+1\right)^{2} \cdot E\left[\mathbf{B}_{k l}\right]}{1-2 E\left[\mathbf{C}_{k l}\right]} \cdot\left(\frac{1}{\hat{N}-N-1}-\frac{1}{\hat{N}-N}\right) \\
\mathbf{V}_{n 5} & =\frac{\left(E\left[\mathbf{C}_{k l}\right]+1\right)^{2}}{1-2 E\left[\mathbf{C}_{k l}\right]} \cdot\left(\frac{1}{\hat{N}-N-1}\right) \\
\mathbf{V}_{d 4} & =\left(1-2 E\left[\mathbf{C}_{k l}\right]\right)\left(E\left[\mathbf{C}_{k l}\right]+1\right)^{2} \\
& -2 E\left[\mathbf{C}_{k l}\right]\left(E\left[\mathbf{C}_{k l}\right]+1\right) E\left[\mathbf{B}_{k l}\right]\left(\frac{1}{\hat{N}-N-1}-\frac{1}{\hat{N}-N}\right) \\
\mathbf{V}_{d 5} & =\frac{E\left[\mathbf{C}_{k l}\right]\left(E\left[\mathbf{C}_{k l}\right]+1\right)}{\hat{N}-N-1}
\end{aligned}
$$

$\mathbf{V}_{n 4}, \mathbf{V}_{n 5}, \mathbf{V}_{d 4}$, and $\mathbf{V}_{d 5}$ are the increased part regardless of newly added user's location and $\left(\frac{d_{2 i}}{d_{1 i}}\right)^{n}+\left(\frac{d_{3 i}}{d_{1 i}}\right)^{n}$ is the increased part depending on the location of the new user. Fig. 3 shows that $\left(\frac{d_{2 i}}{d_{1 i}}\right)^{n}+\left(\frac{d_{3 i}}{d_{1 i}}\right)^{n}$ is the distance of the user from the corresponding BS for $n=4$.

When the added user is located near BS, the increased interference is at minimum, say $\Delta \widehat{\mathbf{I}_{\boldsymbol{\Sigma}}}$. On the contrary the increased interference is at maximum if the user is located equally apart from all three BSs. Then the ratio of the minimum to maximum is

$$
\frac{\Delta \widetilde{\mathbf{I}_{\boldsymbol{\Sigma}} \max }}{\Delta \widetilde{\mathbf{I}_{\boldsymbol{\Sigma}}}}=\left(\frac{\mathbf{V}_{n 4}+2 \mathbf{V}_{n 5}}{\mathbf{V}_{d 4}-2 \mathbf{V}_{d 5}}\right) \cdot\left(\frac{\mathbf{V}_{d 4}}{\mathbf{V}_{n 4}}\right)
$$

As shown in Fig. (4), the ratio decreases with the increase number of users.

\section{Algorithm}

Resource reservation is a method that some users have priority over the others when there are several types of users. If the allocated resource is close to a given threshold, only users with high priority will be accepted by the BS. In the previous section we quantified the resource of multicell CDMA system and found that the interference increases radically as users approach to the cell boundary. If the BS accepts users near the BS instead of far from the BS, naturally we can expect that the network can allow more users into the cell to maximize the throughput by sacrificing the fairness between users. For our convenience we assume a mobile can measure the approximate distance from the BS by the signal power measured through the pilot signal.

In Section III we analyzed how much interference is increased by the addition of a user based on the user's location. The function with respect to the user's location is given by

$$
f\left(d_{1 i}, d_{2 i}, d_{3 i}\right)=\left(\frac{d_{2 i}}{d_{1 i}}\right)^{n}+\left(\frac{d_{3 i}}{d_{1 i}}\right)^{n}
$$

Because the contour near the cell boundary is a close circle as seen in Fig. 3, we can simply write this as $f\left(d_{1}, d_{2 i}, d 3 i\right) \approx$ $f\left(d_{1 i}\right)$. From now on we represent the location of a user by its distance from the corresponding BS.

The notations for the algorithm is given as follows.

- $Z$ : radius of zone-1

- $R E S$ : reserved value of resource

- $r$ : distance between a user and the correspoding BS

- $N_{k, \max }:$ maximum number of active users allowed at sector $k$

We can obtain $N_{\max }$ by using (3).

$$
N_{k, \max }=\frac{1}{S I R_{\min }}+1-\frac{\eta_{o}+I_{o c, k}}{\alpha \cdot r^{n} \cdot P_{\max }}
$$

Here $P_{\max }$ comes from the consideration that the power transmitted by a user is at maximum.

In our algorithm, users are classified as zone-1 (near) and zone-2 (far) users according to their distance from the BS. The purpose of our algorithm is to reserve ' $R E S^{\prime}$ for zone-1 users who are located within the distance $Z$ from the corresponding

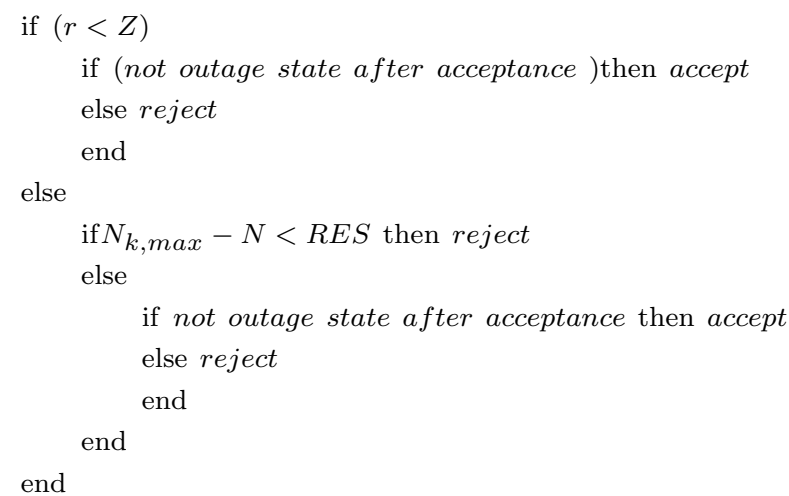

Fig. 5. Two-level zone algorithm. 


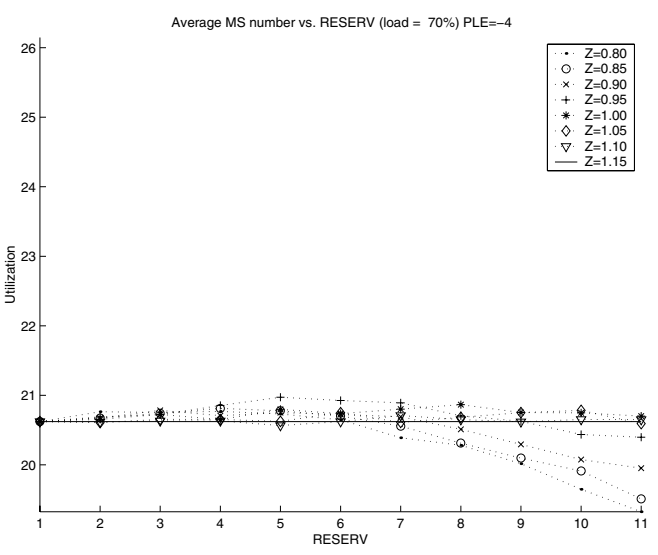

Fig. 6. RES vs. average number of users (load 70\%).

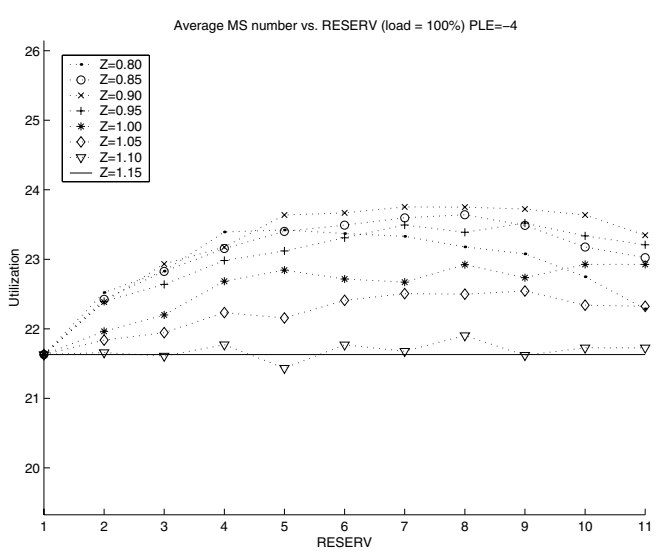

Fig. 7. RES vs. average number of users (load 100\%).

BS. This means that no zone- 2 users who are $Z$ distance away from the BS can be served if $N_{k, \max }-N \leq R E S$ where the number of active users is $N$. The detail algorithm is described in Fig. 5. We call this the two-level zone CAC algorithm.

In this paper we obtain the number of maximum users under the current interference. $N_{k, \max }$ in Fig. 5 is the maximum number of users for the estimated interference. In order to prevent any handoff calls from being dropped due to the increased interference, $\alpha \cdot R^{P L E} \cdot P_{\max }$ is included in (26).

\section{Simulation Results}

For our simulations, we set $E_{b} / N_{0}=6 d B$, and processing gain $=21 d B$. Then the power needs to be controlled to maintain the minimum SIR of $(6-21=-15 d B)$. We assume that the call arrival has Poisson distribution and call duration has exponential distribution with the mean of 1 and the loss path exponent is -4 . By varying $Z, R E S$ and the traffic load, we measured the blocking probability, the average number of users, and the ratio of zone- 1 to zone- 2 user blocking probabilities.

From the simulation results we can find some meaningful results about the two level zone CAC. First, the two-level zone CAC algorithm performs well when the offered load is high. With the increase of load, the average number of

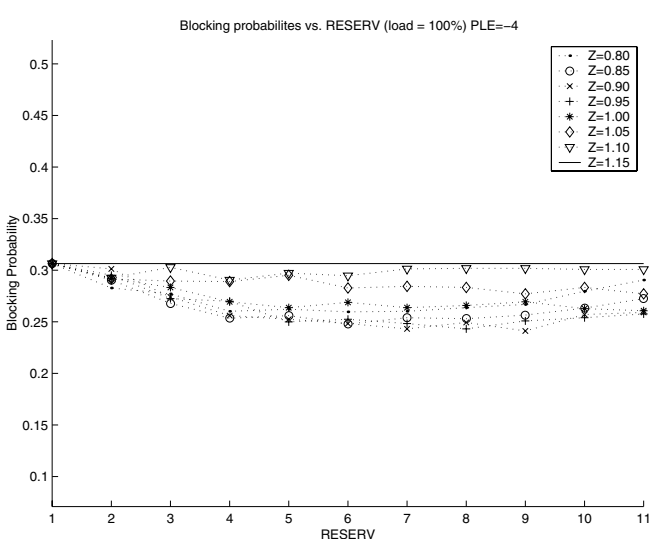

Fig. 8. RES vs. blocking probability (load 100\%).

users increases as shown in Fig. (7). When the load is low, however, performance may be degraded because $R E S$ is so high, resulting in no admission of zone-2 users as shown in Fig. 6. Second, the difference of blocking probabilities of zone-1 and zone- 2 users increases as RES increases. When $R E S$ is large, we can reserve more resources for zone-1 users, accordingly, zone- 2 users get disadvantage. Extremely saying, $R E S=\hat{N}$ means that the resource is reserved for only zone-1 users, i.e., zone-2 users suffer from heavy blocking. Reversely $R E S=0$ means that the two-level zone CAC just runs as an ordinary algorithm.

To find an appropriate $R E S$ we need to consider the tradeoff relationship between throughput improvement and fairness. For large $Z$, the results show a small difference in performance because the controlled area is small. On the contrary too small $Z$ may result in the degradation of performance because a user requesting small resource may be blocked. In Fig. 8 the blocking probability shows a symmetry when compared with the average number of users as seen in Fig. 7. The difference in the blocking probability increases for high $R E S$ and low $Z$ as shown in Fig. 9.

\section{CONCLUSION}

In this paper we modeled a cell by representing it as a $3 \frac{1}{6}$ cell, and exactly quantified the channel capacity for multicell CDMA environments. We considered only the effect of path loss for simplicity. Through the analysis we verified that users near the cell boundary generate more noise than those close to the BS. By using the fact that the number of users supported by a cell depends on the location of each user, we proposed the two-level CAC algorithm that divides the cell area into two zones (near and far zones from the BS) and gives priority to users near the BS. We showed that the total interference can be represented by a user's distance from the BS. By allowing more near users into the network, we could utilize the cell capacity at maximum. Simulation results showed that RES and $Z$ parameters are used for the optimal division of two zones.

The cost to implement this idea is losing some fairness between near and far users from the BS. So there exists 


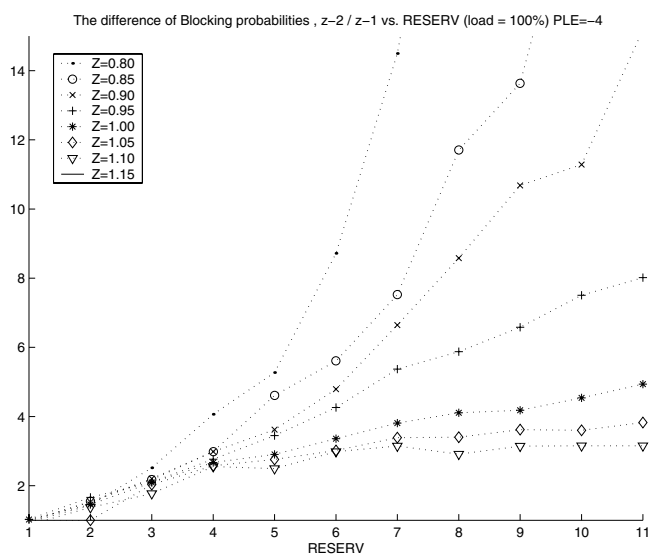

Fig. 9. RES vs. the ratio of blocking probability in zone1, 2 (load 100\%).

a trade off betwen the increased number of admitted users and the degree of losing fairness. Also our scheme has a disadvantage of increased complexity in deciding whether a user is near or far from the BS according to the measured pilot signal strength. However, our algorithm is easily applicable to cellular networks by a handoff scheme because the handoff region divides cellular coverage into two zones. Additively the benefit of increased capacity can be used for handoff calls by channel reservation, thereby reducing handoff dropping.

\section{REFERENCES}

[1] J. S. Lee and L. E.Miller, "CDMA Systems Engineering Handbooks" Artech House Publishers, Boston USA, 1998.

[2] Z. Liu and M. E. Zarki, "SIR-based Call Admission Control for DS-CDMA Cellular Systems" IEEE Journal on Selected Areas in Communications, Vol.12, May 1994.

[3] S. A. Grandhi, R. D. Yates, and D. J. Goodman, "Resource Allocation for Cellular Radio Systems" IEEE Transactions on Vehicular Technology, Vol. 46, Aug 1997.

[4] K. S. Gilhousen, I. Jacobs, R. Padovani, A. Viterbi, L. A.Weaver. Jr. and Charles E.Wheatley III, "On the Capacity of a Cellular CDMA System" IEEE Transactions on Vehicular Technology, Vol. 40, No. 2, May 1991.

[5] K. Kim, "CDMA Cellular Engineering Issues", IEEE Transactions on Vehicular Technology, Vol. 42, No. 3, Aug 1993.

[6] G. L. Stueber and C. Kchao, "Analysis of a Multiple-cell Direct-Sequence CDMA Cellular Mobile Radio System" IEEE Journal on Selected Areas in Communications, Vol. 10, pp. 669-679, May 1992.

[7] C. Oliveira, J. B. Kim and T. Suda, "An Adaptive Bandwidth Reservation Scheme for High-speed Multimedia Wireless Networks" IEEE Journal on Selected Areas in Communications, Vol. 16, pp. 858-874, Aug 1998.

[8] A. Arregui and J. Dunlop, "Distributed Call Admission Control in Partially Loaded TDMA Systems" IEEE Vehicular Technology Conference (VTC), 1998. 